\title{
A database of genus-2 curves over the rational numbers
}

\author{
Andrew R. Booker, Jeroen Sijsling, Andrew V. Sutherland, \\ John Voight and Dan Yasaki
}

\begin{abstract}
We describe the construction of a database of genus- 2 curves of small discriminant that includes geometric and arithmetic invariants of each curve, its Jacobian, and the associated $L$-function. This data has been incorporated into the $L$-Functions and Modular Forms Database (LMFDB).
\end{abstract}

\section{Introduction}

The history of computing tables of elliptic curves over the rational numbers extends as far back as some of the earliest machine-aided computations in number theory. The first of these tables appeared in the proceedings of the 1972 conference held in Antwerp [3]. Vast tables of elliptic curves now exist, as computed by Cremona [12-14] and Stein and Watkins [45]. These tables have been used extensively in research in arithmetic geometry to test and formulate conjectures, and have thereby motivated many important advances.

In this article, we continue this tradition by constructing a table of genus- 2 curves over the rational numbers. We find a total of 66158 isomorphism classes of curves with absolute discriminant at most $10^{6}$; for each curve, we compute an array of geometric and arithmetic invariants of the curve and its Jacobian, as well as information about rational points and the associated $L$-function. This data significantly extends previously existing tables (see $\S 3.4$ for a comparison and discussion of completeness), and it is available online at the $L$-functions and Modular Forms Database (LMFDB) [34]. We anticipate that the list of curves and the auxiliary data we have computed will be useful in ongoing investigations in genus 2, including the Sato-Tate conjecture as formulated by Fité et al. [18] and the paramodular conjecture of Brumer and Kramer [8].

This article is organized as follows. In $\S 2$ we set up basic notation and background. In $\S 3$ we describe our search procedure. In $\S \S 4-6$ we outline the computation of various invariants associated to the curve and its Jacobian. Finally, in the Appendix, we provide tables exhibiting some interesting aspects of the data.

\section{Notation and background}

In this section, we set up basic notation and recall some relevant background material. For a survey of computational aspects of genus- 2 curves, see the article by Poonen [39].

Received 11 February 2016.

2010 Mathematics Subject Classification 11G30, 14H45, 14Q05 (primary).

Contributed to the Twelfth Algorithmic Number Theory Symposium (ANTS-XII), Kaiserslautern, Germany, 29 August-2 September 2016.

The first author was supported by EPSRC grants EP/H005188/1, EP/L001454/1 and EP/K034383/1. The third author was supported by NSF grants DMS-1115455 and DMS-1522526. The fourth author was supported by an NSF CAREER Award (DMS-1151047). The fifth author was partially supported by NSA grant H98230-15-1-0228. 
Let $A$ be an abelian variety over a field $F$. For a field extension $K \supseteq F$, we denote by $A_{K}$ the abelian variety over $K$ obtained from $A$ by base extension from $F$ to $K$. We write $\operatorname{End}(A)$ to denote the ring of endomorphisms of $A$ defined over $F$; we write $\operatorname{End}\left(A_{K}\right)$ for endomorphisms of $A$ defined over an extension $K$. For a $\mathbb{Z}$-algebra $R$ we use $\operatorname{End}(A)_{R}:=\operatorname{End}(A) \otimes_{\mathbb{Z}} R$ to denote the $R$-endomorphism algebra of $A$. Finally, by a curve over a field $F$ we mean a smooth, projective, geometrically integral scheme of finite type over $F$ of dimension 1.

\subsection{Models and discriminants}

Let $F$ be a perfect field and let $X$ be a curve of genus 2 over $F$. Then $X$ has a Weierstrass model (or Weierstrass equation)

$$
y^{2}+h(x) y=f(x),
$$

where $f, h \in F[x]$ have degrees bounded by 6 and 3 , respectively. It follows that $X$ is hyperelliptic over $F$, with the map $x: X \rightarrow \mathbb{P}^{1}$ of degree 2 .

Now suppose that char $F \neq 2$. Completing the square yields a simplified Weierstrass model $y^{2}=4 f(x)+h(x)^{2}$ for $X$. For $n \geqslant 1$, let $g_{n, \text { univ }}(x)=a_{n} x^{n}+\ldots+a_{1} x+a_{0} \in \mathbb{Z}\left[a_{0}, \ldots, a_{n}\right][x]$ and define

$$
\operatorname{disc}_{n}:=\frac{1}{a_{n}}(-1)^{n(n-1) / 2} \operatorname{Res}\left(g_{n, \text { univ }}(x), \frac{d}{d x} g_{n, \text { univ }}(x)\right) \in \mathbb{Z}\left[a_{0}, \ldots, a_{n}\right] .
$$

For a polynomial $g \in F[x]$ with $\operatorname{deg}(g) \leqslant n$, define $\operatorname{disc}_{n}(g) \in F$ by specialization. Following Liu [31], the discriminant of the Weierstrass model $y^{2}+h(x) y=f(x)$ is defined by

$$
\Delta(f, h):=2^{-12} \operatorname{disc}_{6}\left(4 f+h^{2}\right) .
$$

For $f_{\text {univ }}(x)=\sum_{i=0}^{6} f_{i} x^{i}$ and $h_{\text {univ }}(x)=\sum_{i=0}^{3} h_{i} x^{i}$ in $\mathbb{Z}\left[f_{0}, \ldots, f_{6}, h_{0}, \ldots, h_{3}\right][x]$, the greatest common divisor of the coefficients of $\operatorname{disc}_{6}\left(4 f_{\text {univ }}+h_{\text {univ }}^{2}\right)$ is $2^{12}$, so

$$
\Delta\left(f_{\text {univ }}, h_{\text {univ }}\right) \in \mathbb{Z}\left[f_{0}, \ldots, f_{6}, h_{0}, \ldots, h_{3}\right],
$$

and in particular, if $f, h \in R[x]$, then $\Delta(f, h) \in R$ for a domain $R \subseteq F$. For any $f, h \in F[x]$ with $\operatorname{deg} f \leqslant 6$ and $\operatorname{deg} h \leqslant 3$, we have $\Delta(f, h) \neq 0$ if and only if $y^{2}+h(x) y=f(x)$ is a Weierstrass model for a genus- 2 curve over $F$. In the special case where $f$ is monic with $\operatorname{deg}(f)=5$ and $\operatorname{deg} h \leqslant 2$ (which can be achieved if and only if $X$ has a Weierstrass point defined over $F$ ), this agrees with the definition given by Lockhart [35].

If $\left(y^{\prime}\right)^{2}+h^{\prime}\left(x^{\prime}\right) y^{\prime}=f^{\prime}\left(x^{\prime}\right)$ is another Weierstrass model for the curve $X$, then it is related to (2.1.1) via a change of variables of the form

$$
x^{\prime}=\frac{a x+b}{c x+d}, \quad y^{\prime}=\frac{e y+j(x)}{(c x+d)^{3}}, \quad \text { with } a d-b c, e \in F^{\times}, j \in F[x], \operatorname{deg} j \leqslant 3,
$$

and we have $\Delta\left(f^{\prime}, h^{\prime}\right)=e^{20}(a d-b c)^{-30} \Delta(f, h)$; see Liu [31, $\left.\S 1.3\right]$. Thus, as an element of $F^{\times} / F^{\times 10}$, the discriminant $\Delta(f, h)$ is an invariant of $X$, and if $v$ is a discrete valuation on $F$, then $v(\Delta(f, h)) \bmod 10$ is also an invariant.

Now suppose that $F$ is a local field or a (global) number field, and let $R$ be the ring of integers of $F$. An integral model of $X$ is a Weierstrass model (2.1.1) with $f, h \in R[x]$, which implies $\Delta(f, h) \in R$. We may rescale any given Weierstrass model to obtain an integral model after clearing denominators. If $F$ is a non-archimedean local field with discrete valuation $v$, then a minimal model for $X$ is an integral model that minimizes $v(\Delta(f, h)) \in \mathbb{Z}_{\geqslant 0}$, and the 
minimal discriminant $\Delta_{\min }(X)$ is the $R$-ideal generated by $\Delta(f, h)$ for any minimal model $y^{2}+h(x) y=f(x)$. If instead $F$ is a number field, then the minimal discriminant of $X$ is defined by

$$
\Delta_{\min }(X):=\prod_{\mathfrak{p}} \Delta_{\min }\left(X_{F_{\mathfrak{p}}}\right) \subseteq R
$$

where $\mathfrak{p}$ ranges over the non-zero prime ideals of $R$ and $F_{\mathfrak{p}}$ is the completion of $F$ at $\mathfrak{p}$, and we say that an integral model for $X$ is a global minimal model if $\Delta(f, h) R=\Delta_{\min }(X)$. Global minimal models do not always exist, but if $F$ has class number 1, then $X$ has a global minimal model [33, Remarque 6]. Minimal models for genus-2 curves over $\mathbb{Q}$ can be computed in Magma [6] via the function MinimalWeierstrassModel, implemented by Michael Stoll.

When $F=\mathbb{Q}$, the discriminant $\Delta(f, h) \in \mathbb{Z}$ of every minimal model is the same, since it is determined up to sign by the principal ideal $\Delta_{\min }(X)$ and $-1 \notin \mathbb{Q}^{\times 10}$; we then view $\Delta_{\min }(X)$ as the integer $\Delta(f, h) \in \mathbb{Z}$ and define the absolute discriminant of $X$ to be $\left|\Delta_{\min }(X)\right| \in \mathbb{Z}_{>0}$.

\section{Searching in a box}

We wish to enumerate integral models $y^{2}+h(x) y=f(x)$ of genus-2 curves $X$ over $\mathbb{Q}$ with the goal of finding as many non-isomorphic curves as possible with absolute discriminant $\left|\Delta_{\min }(X)\right| \leqslant D$, where $D:=10^{6}$ is our chosen discriminant bound. Computing the minimal discriminant of a given curve $X$ is time-consuming, so we instead just search for integral models with $|\Delta(f, h)| \leqslant D$. We then minimize these models if necessary, but this is rarely required; absolute discriminants bounded by $10^{6}$ will necessarily have minimal valuation at all primes $p \geqslant 5$, since $5^{10}>10^{6}$. Our approach is to enumerate a large finite set $S$ of integral models from which we will select those with $|\Delta(f, h)| \leqslant D$. The set $S$ is defined by constraints on the coefficients of $f, h \in \mathbb{Z}[x]$ that are chosen so that $S$ contains many minimal models.

\subsection{The shape of the box}

We can assume without loss of generality that the coefficients $h_{i}$ of $h(x)=\sum_{i=0}^{3} h_{i} x^{i}$ lie in the set $\{0,1\}$ : indeed, if $h^{\prime} \equiv h(\bmod 2)$, applying $(2.1 .2)$ with $j=\left(h^{\prime}-h\right) / 2$ and $e=1$ and $x^{\prime}=x$ yields an integral model $\left(y^{\prime}\right)^{2}+h^{\prime}(x) y^{\prime}=f^{\prime}(x)$ with the same discriminant. We cannot, however, assume $h=0$; indeed, only a small fraction of the curves we eventually found have minimal models with $h=0$. This leaves 16 possible $h \in \mathbb{Z}[x]$ with $\operatorname{deg} h \leqslant 3$ to consider. One can reduce this to six via the substitutions $x \mapsto 1 / x$ and $x \mapsto x+1$ which leave $\Delta(f, h)$ fixed; however, we choose not to do this as these transformations may move a model outside the set $S$ we are searching (we do use this symmetry to optimize our search).

For the coefficients of $f=\sum_{0}^{6} f_{i} x^{i}$ we impose constraints of the form $\left|f_{i}\right| \leqslant B_{i}$ that determine the shape of a box (orthogonal parallelepiped) in which we hope to find integral models with small discriminants. We experimented with various shapes and eventually settled on three:

$$
\begin{aligned}
S_{1}(B) & :=\left\{(f, h):\left|f_{i}\right| \leqslant B, h_{i}=0,1\right\}, \\
S_{2}(a, b) & :=\left\{(f, h):\left|f_{i}\right| \leqslant a b^{6-i}, h_{i}=0,1\right\}, \\
S_{3}(b) & :=\left\{(f, h):\left|f_{i}\right| \leqslant b^{4-|i-3|}, h_{i}=0,1\right\} .
\end{aligned}
$$

The 'flat' box $S_{1}$ is a hypercube (a ball in the $L^{\infty}$-metric). The 'weighted' box $S_{2}$ complements $S_{1}$ and tries to find curves with small discriminant but large 'flat' height, with weighting designed to give each term in the discriminant roughly the same size. The 'hill' box $S_{3}$ complements $S_{1}, S_{2}$ and tries to find curves with small discriminant but large 'flat' and 'weighted' height: it allows larger middle coefficients with smaller end coefficients. 
Taken together, the boxes $S_{i}$ are a crude attempt to take the oddly shaped region defined by $\operatorname{disc}(f, h) \leqslant X$ and approximate it with boxes that have little overlap. Table A.1 lists the number of isomorphism classes with $|\Delta(f, h)| \leqslant 10^{n}$ for $n=3,4,5,6$, using boxes of cardinality $10^{m}$ with $m=11, \ldots, 17$ for these three shapes.

To squeeze out a bit more, we also considered sets

$$
S_{4}(b, d):=\left\{(f, h): \sum_{i}\left\lceil\log _{b}\left(\left|f_{i}\right|+1\right)\right\rceil \leqslant d, h_{i}=0,1\right\},
$$

which can be viewed as a union of $\left(\begin{array}{c}d+6 \\ 6\end{array}\right)$ boxes. For our final search we settled on

$$
S_{1}:=S_{1}(90), \quad S_{2}:=S_{2}(2,3.51), \quad S_{3}:=S_{3}(7.14), \quad S_{4}:=S_{4}(10,10),
$$

with cardinalities $\# S_{1}, \# S_{2}, \# S_{3} \approx 10^{17}$ and $S_{4} \approx 10^{16.3}$; see Table A.2 for more details.

\subsection{Computing discriminants}

The discriminant polynomial $\operatorname{disc}_{6} \in \mathbb{Z}\left[a_{0}, \ldots, a_{6}\right]$ is a homogeneous polynomial of degree 10 with 246 terms. Directly computing $\Delta(f, h)=2^{-12} \operatorname{disc}_{6}\left(4 f+h^{2}\right)$ for a single model $(f, h)$ involves at least 1000 arithmetic operations, but to compute $\Delta(f, h)$ for all $(f, h)$ in a box we instead use an algorithm that efficiently enumerates values of a multivariate polynomial $g\left(x_{1}, \ldots, x_{n}\right)$ over integer points in a box $A_{1} \times \ldots \times A_{n}$ of $\mathbb{Z}^{n}$. With this approach we require less than six clock cycles, on average, to compute each discriminant $\Delta(f, h)$ for $(f, h) \in S$.

The algorithm first creates a monomial tree with $n$ levels. A node at level $m \leqslant n$ in the tree corresponds to a monomial in $x_{1}, \ldots, x_{m}$, identified by a vector of $m$ non-negative exponents, and contains storage for an integer coefficient. The nodes at level $n$ (the leaves) correspond to the terms of $g$. The nodes at levels $m<n$ correspond to the terms of a partially instantiated polynomial $g\left(x_{1}, \ldots, x_{m}, a_{m+1}, \ldots, a_{n}\right)$, for some $a_{m+1}, \ldots, a_{n} \in \mathbb{Z}$; the monomial exponents depend only on $g$, but the coefficients vary with $a_{m+1}, \ldots, a_{n}$. Each node at level $m+1$ has an edge leading to the node at level $m$ corresponding to the substitution $x_{m+1}=a_{m+1}$.

For $n=1$, the polynomial $g\left(x_{1}\right)$ is univariate, and we may efficiently enumerate its values at integers in the interval $A_{1}$ using finite differences, as in Kedlaya and Sutherland [29, $\S 3$ ] Each step requires just $d$ additions, where $d=\operatorname{deg}_{x_{1}} g$. For $n>1$ we reduce to the case $n-1$ as follows. We begin with $a_{n}=\min \left(A_{n}\right)$ and compute the powers $a_{n}^{i}$ for $0 \leqslant i \leqslant d$, where $d=\operatorname{deg}_{x_{n}} g$. We then use these powers to instantiate the monomials at level $n$ of the tree at $x_{n}=a_{n}$, using one multiplication per node, thereby obtaining a coefficient value for each instantiated monomial. We then set the coefficient value of each node at level $n-1$ to the sum of the coefficient values of its instantiated children. The nodes at level $n-1$ now represent the terms of the polynomial $g\left(x_{1}, \ldots, x_{n-1}, a_{n}\right)$ in $n-1$ variables whose values we may recursively enumerate over integer points in $A_{1} \times \ldots \times A_{n-1}$. We then increment $a_{n}$ and repeat until $a_{n}>\max \left(A_{n}\right)$.

The time to enumerate the values of $g\left(x_{1}, \ldots, x_{n}\right)$ over $A_{1} \times \ldots \times A_{n}$ is dominated by the time spent in the bottom layer of the recursion $(n=1)$, which involves $\operatorname{deg}_{x_{1}} g$ integer additions. We treat each of the 16 possible values of $h$ as fixed, and for each $h$ view $\operatorname{disc}_{6}\left(4 f_{\text {univ }}+h^{2}\right)$ as a polynomial in the $n=7$ variables $f_{0}, \ldots, f_{6}$. Using a monomial tree to enumerate values of $g:=\operatorname{disc}_{6}\left(4 f_{\text {univ }}+h^{2}\right) \in \mathbb{Z}\left[f_{0}, \ldots, f_{6}\right]$ yields an algorithm that essentially uses just $\operatorname{deg}_{f_{0}} g=$ 5 additions per discriminant. As a further optimization, we work modulo $2^{64}$ so that each addition can be accomplished in a single clock cycle. The monomial tree for $g$ contains 703 nodes and requires less than $8 \mathrm{kB}$ of storage, which easily fits in the L1 data cache of a modern CPU.

To find integral models $y^{2}+h(x) y=f(x)$ with small discriminant, we look for pairs $(f, h)$ for which $\Delta(f, h) \equiv \Delta\left(\bmod 2^{64}\right)$ for some integer $|\Delta| \leqslant D$. For the relatively few pairs that pass 
this test, we recompute $\Delta(f, h)$ over $\mathbb{Z}$ to check if in fact $|\Delta(f, h)| \leqslant D$; this recomputation occurs so rarely that it has a negligible impact on the total running time.

\subsection{Parallel computation}

Our search can be trivially parallelized by partitioning each box into products of the form $A_{1} \times \ldots \times A_{n}$ and applying the algorithm described in the previous section. Using the compute engine on Google's Cloud Platform [23], we ran a parallel computation to enumerate all pairs $(f, h)$ with $|\Delta(f, h)| \leqslant 10^{6}$ in the sets $S_{1}, S_{2}, S_{3}, S_{4}$ defined above, which together contain over $3 \times 10^{17}$ pairs $(f, h)$. We allocated 2250 high-CPU compute nodes distributed across six of Google's compute engine regions (two in the central USA, three in the eastern USA, and one in western Europe). Each high-CPU node comes equipped with 32 Intel Haswell cores and $28.8 \mathrm{~GB}$ of memory, yielding a total of 72000 cores. We performed approximately 50 core-years of computation over the course of less than 8 hours of elapsed time. To reduce the cost we used preemptible nodes. On average, roughly $3 \%$ of our nodes were preempted each hour; these were automatically restarted from checkpoints that were created every few minutes.

\subsection{Completeness}

We now compare our results with existing tables of genus-2 curves over $\mathbb{Q}$. In every case we find that our tables include all previously known examples that fall within our discriminant range (as well as many that are completely new).

- The most comprehensive list of genus-2 curves over $\mathbb{Q}$ previously compiled is due to Stoll [46], who searched for curves with odd absolute discriminants bounded by $11^{4}$, finding 823 distinct $\mathbb{Q}$-isomorphism classes with 749 distinct discriminants. Our search found all of these curves, as well as many more: in total, we found $988 \mathbb{Q}$-isomorphism classes of genus- 2 curves over $\mathbb{Q}$ with 852 distinct odd discriminants of absolute value up to $11^{4}$. Our tables differ already at the third smallest absolute minimal discriminant $\left|\Delta_{\min }(X)\right|=277$, where we find two non-isomorphic curves

$$
\begin{gathered}
y^{2}+\left(x^{3}+x^{2}+x+1\right) y=-x^{2}-x, \\
y^{2}+y=x^{5}-9 x^{4}+14 x^{3}-19 x^{2}+11 x-6,
\end{gathered}
$$

only one of which (the first) was found by Stoll [46]. The smallest odd absolute discriminant found in our search that is new is 1665 , which arises for the curve

$$
y^{2}+\left(x^{3}+x^{2}+1\right) y=x^{4}+x^{3}+2 x^{2}+2 x+1 .
$$

- Merriman and Smart $[36,42]$ determined all genus-2 curves over $\mathbb{Q}$ with good reduction away from 2. Up to $\mathbb{Q}$-isomorphism there are 427 such curves, of which 29 curves have absolute discriminant bounded by $10^{6}$, all found by our search. (The models given by Smart [42, Table] are not necessarily minimal; we used Magma [6] to compute minimal models.)

- Baker et al. [1] and González et al. [21] determined a complete list of new modular curves of genus 2, that is, those curves $X$ that admit a dominant morphism $\pi: X_{1}(N) \rightarrow X$ from the modular curve $X_{1}(N)$ (for which $\pi^{*}(\operatorname{Jac}(X)) \subseteq J_{1}(N)_{\text {new }}$ ) for some level $N \in \mathbb{Z}_{\geqslant 1}$. Up to $\mathbb{Q}$-isomorphism there are 213 such curves: 149 have $\mathbb{Q}$-simple Jacobians [21] and 64 have split Jacobians [1]. Of these 213 curves, all 61 with absolute discriminant bounded by $10^{6}$ were found in our search.

- González et al. [22] (see also González et al. [19]) computed equations of genus-2 curves whose Jacobians are modular abelian varieties, that is, they are subabelian varieties of the Jacobian $J_{1}(N)$ for some $N \in \mathbb{Z}_{\geqslant 1}$. Among the 75 curves they list, the three with absolute discriminant bounded by $10^{6}$ were found in our search. 
- González and Rotger [20] computed explicit models for some Shimura curves of genus 2 ; of these, the six curves with absolute discriminant bounded by $10^{6}$ were also found in our search.

- In pursuit of the paramodular conjecture, Brumer and Kramer [8] list 38 genus-2 curves $X$ whose $\operatorname{Jacobians} \operatorname{Jac}(X)$ have $\operatorname{End}(\operatorname{Jac}(X))=\mathbb{Z}$ of odd conductor less than 1000 . Of these, 33 have absolute discriminant bounded by $10^{6}$, all of which were found in our search. In total we found $56 \mathbb{Q}$-isomorphism classes of genus- 2 curves $X$ whose Jacobians have odd conductor less than 1000 , of which 43 curves have $\operatorname{End}(\operatorname{Jac}(C))=\mathbb{Z}$. These all appear to lie in the 33 isogeny classes identified by Brumer and Kramer using Jacobians of curves with absolute discriminants below $10^{6}$.

- In a similar vein, Farmer et al. [17] used analytic methods to determine a complete list of the integers $N \leqslant 500$ that can arise as the conductor of degree- $4 L$-functions under a standard set of hypotheses. Excluding $L$-functions arising from products of elliptic curves or classical modular forms with real quadratic character, they list 12 values of $N \leqslant 500$ that may arise for the $L$-function of a genus-2 curve [17, Theorem 2.1] (the nine odd values match the Brumer-Kramer list up to 500). We found 21 curves matching these 12 values (and at least one for each $N$ ), including six curves that match the even values $388,464,472$ on their list.

Despite our attempts at exhaustiveness, the data in Table A.1 suggest that our tables are still incomplete. Indeed, there are genus- 2 curves with absolute minimal discriminant bounded by $10^{6}$ that do not have an integral model in any of the sets $S_{1}, S_{2}, S_{3}, S_{4}$ that we searched: we found 28 such curves while experimenting with boxes of different shapes. One way to get a sense for the completeness of our tables is to consider an analogous search among genus- 1 curves. The discriminant polynomial then has degree 6 , rather than 10 , so we use the discriminant bound $\left\lfloor\left(10^{6}\right)^{6 / 10}\right\rfloor=3981$. The Jacobian of such a genus- 1 curve is isomorphic to an elliptic curve whose conductor must also be bounded by 3981 and can therefore be found in Cremona's tables [14] (by Ogg's formula, the conductor of an elliptic curve over $\mathbb{Q}$ divides its minimal discriminant). In total there are $1154 \mathbb{Q}$-isomorphism classes of elliptic curves with absolute discriminant bounded by 3981. A search for genus-1 curves $y^{2}+h(x) y=f(x)$ with $\operatorname{deg} h \leqslant 2$ and $\operatorname{deg} f \leqslant 4$ using the same coefficient bounds as in the box $S_{1}(90)$ already hits 1132 of these $\mathbb{Q}$-isomorphism classes, or about $98 \%$.

\section{Computing basic data}

\subsection{Invariants}

The isomorphism class of a genus- 2 curve over an algebraically closed field can be characterized by a number of invariants. For elliptic curves, only the elliptic $j$-invariant is required. In genus 2 , there are different choices of invariants available, all with their own distinct advantages and drawbacks. We refer to seminal work by Igusa [27] and later work by Mestre [37] for some theoretical expositions. The details of the implementation in MAGMA can be found in its Handbook [5]; the package was written by Everett Howe, based on routines developed by Fernando Rodriguez-Villegas, and with additional functionality added by Reynald Lercier and Christophe Ritzenthaler.

Let $F$ be a field with char $F \neq 2$ and let $X$ be a genus- 2 curve over $F$. Then $X$ has a simplified Weierstrass model

$$
X: y^{2}=f(x)=c \prod_{i=1}^{6}\left(x-\alpha_{i}\right)
$$

where the $\alpha_{i}$ are the distinct roots of $f$ in an algebraic closure $\bar{F}$ of the base field $F$. Given two such roots $\alpha_{i}, \alpha_{j}$, we denote the difference $\alpha_{i}-\alpha_{j}$ by $(i j)$. We then form the 
expressions

$$
\begin{aligned}
I_{2} & :=(4 c)^{2} \sum(12)^{2}(34)^{2}(56)^{2}, \\
I_{4} & :=(4 c)^{4} \sum(12)^{2}(23)^{2}(31)^{2}(45)^{2}(56)^{2}(64)^{2}, \\
I_{6} & :=(4 c)^{6} \sum(12)^{2}(23)^{2}(31)^{2}(45)^{2}(56)^{2}(64)^{2}(14)^{2}(25)^{2}(36)^{2}, \\
I_{10} & :=(4 c)^{10} \prod(12)^{2}=\operatorname{disc}_{6}(4 f),
\end{aligned}
$$

where each sum and product runs over the distinct expressions obtained by permuting the index set $\{1, \ldots, 6\}$. The invariants $I_{2}, I_{4}, I_{6}, I_{10}$, defined by Igusa $[\mathbf{2 7}$, p. 620] by modifying a set of invariants due to Clebsch, are known as the Igusa-Clebsch invariants. Two curves $X$ and $X^{\prime}$ over $F$ are isomorphic over the algebraic closure $\bar{F}$ if and only if

$$
\left(I_{2}: I_{4}: I_{6}: I_{10}\right)=\left(I_{2}^{\prime}: I_{4}^{\prime}: I_{6}^{\prime}: I_{10}^{\prime}\right) \in \mathbb{P}(2,4,6,10)(\bar{F})
$$

describe the same point in weighted projective $(2,4,6,10)$-space; that is, if and only if there exists a $\lambda \in \bar{F}^{\times}$such that $\left(I_{2}^{\prime}, I_{4}^{\prime}, I_{6}^{\prime}, I_{10}^{\prime}\right)=\left(\lambda^{2} I_{2}, \lambda^{4} I_{4}, \lambda^{6} I_{6}, \lambda^{10} I_{10}\right)$.

The Igusa-Clebsch invariants break down in characteristic 2 , and to deal with this problem, Igusa [27, pp. 617ff.] found a common normal form for hyperelliptic curves in arbitrary characteristic. Motivated by this, he defined the invariants [27, pp. 621-622]

$$
\begin{aligned}
J_{2} & :=I_{2} / 8 \\
J_{4} & :=\left(4 J_{2}^{2}-I_{4}\right) / 96, \\
J_{6} & :=\left(8 J_{2}^{3}-160 J_{2} J_{4}-I_{6}\right) / 576, \\
J_{8} & :=\left(J_{2} J_{6}-J_{4}^{2}\right) / 4, \\
J_{10} & :=I_{10} / 4096,
\end{aligned}
$$

now called the Igusa invariants, with $\left(J_{2}: J_{4}: J_{6}: J_{8}: J_{10}\right) \in \mathbb{P}(2,4,6,8,10)(\bar{F})$. Moreover, these invariants are compatible with specialization of the model, for example, reducing modulo a prime.

While the Igusa invariants are in a sense the best available with respect to arithmetic questions, we need five of them to describe a moduli space of dimension 3 . However, the moduli space of genus- 2 curves over a given field admits a simpler description: the corresponding moduli space can always be described by three affine coordinates. Such coordinates were given by Cardona et al. $[\mathbf{1 0}, \mathbf{1 1}]$. For $\operatorname{char} F \neq 2$, these 62 -invariants (sometimes also known as absolute Igusa invariants) can be defined by

$$
\left(g_{1}, g_{2}, g_{3}\right)= \begin{cases}\left(J_{2}^{5} / J_{10}, J_{2}^{3} J_{4} / J_{10}, J_{2}^{2} J_{6} / J_{10}\right) & \text { if } J_{2} \neq 0 \\ \left(0, J_{4}^{5} / J_{10}^{2}, J_{4} J_{6} / J_{10}\right) & \text { if } J_{2}=0, J_{4} \neq 0 \\ \left(0,0, J_{6}^{5} / J_{10}^{3}\right) & \text { otherwise }\end{cases}
$$

The G2-invariants are absolute, with $\left(g_{1}, g_{2}, g_{3}\right) \in \mathbb{A}^{3}(F)$ in affine space. This time, $X$ has potentially good reduction at a prime if and only if none of the G2-invariants reduce to $\infty$ modulo that prime. Conversely, given three G2-invariants in a field $k$, there always exists a curve with these invariants. However, writing down such a curve may require a quadratic extension of $k$. This obstruction was studied by Mestre [37] and by Cardona and Quer [11].

\section{2. $L$-factors at good primes}

The $L$-series of a genus-2 curve $X$ over $\mathbb{Q}$ is a Dirichlet series with an Euler product

$$
L(X, s)=\sum_{n=1}^{\infty} \frac{a_{n}}{n^{s}}=\prod_{p} L_{p}\left(p^{-s}\right)
$$


such that for primes $p$ of good reduction we have

$$
Z\left(X / \mathbb{F}_{p}, T\right)=\exp \left(\sum_{r=1}^{\infty} \# X\left(\mathbb{F}_{p^{r}}\right) \frac{T^{r}}{r}\right)=\frac{L_{p}(T)}{(1-T)(1-p T)}
$$

and $L_{p}(T) \in 1+T \mathbb{Z}[T]$ has degree 4. Efficient methods for computing the coefficients of $L_{p}(T)$ for all good primes $p$ up to a given bound are discussed in Kedlaya and Sutherland [29] and by Harvey and Sutherland $[\mathbf{2 4}, \mathbf{2 5}]$ and available in smalljac [44].

\subsection{Hash function}

If abelian varieties $A, A^{\prime}$ over $\mathbb{Q}$ are isogenous over $\mathbb{Q}$, then $a_{p}(A)=a_{p}\left(A^{\prime}\right)$ for all primes $p$. To facilitate the efficient grouping of curves into candidate isogeny classes (of their Jacobians), we define the hash of an abelian surface $A$ to be the unique integer $h(A) \in[0, P-1]$ for which

$$
h(A) \equiv \sum_{\substack{2^{12}<p<2^{13} \\ p \text { prime }}} c_{p} a_{p}(A) \bmod P,
$$

where $P=2^{61}-1$ and $c_{p}=\left\lfloor\pi P^{e_{p}}\right\rfloor$, with $e_{p}:=\#\left\{q: 2^{12}<q \leqslant p, q\right.$ prime $\}$ and $\pi=3.14 \ldots$ (so the $c_{p}$ correspond to successive digits in the base- $P$ expansion of $\pi$ ). Isogenous abelian surfaces necessarily have the same hash, and while the converse need not hold in general, it almost always does. Hashes also provide a way to provisionally identify automorphic objects (such as modular forms) that have the same $L$-function.

\subsection{Sato-Tate group}

For an abelian surface $A$ over a number field $F$, there are 52 distinct Sato-Tate groups $\operatorname{ST}(A)$ that arise, of which 34 occur when $F=\mathbb{Q}[\mathbf{1 8}$, Theorem 1.5]. A coarser classification is obtained if we restrict our attention to the identity component $\operatorname{ST}\left(A^{0}\right)$, or equivalently, the Sato-Tate group $\mathrm{ST}\left(A_{K}\right)$ of the base extension of $A$ to the minimal field $K$ over which all its endomorphisms are defined. In this case there are just six possibilities, corresponding to six types of real endomorphism algebras $\operatorname{End}\left(A_{K}\right)_{\mathbb{R}}:=\operatorname{End}\left(A_{K}\right) \otimes_{\mathbb{Z}} \mathbb{R}$, all of which can arise when $F=\mathbb{Q}$. These are listed in Table 1 , along with the possible types of abelian varieties $A_{K}$ (up to isogeny).

Under the generalized Sato-Tate conjecture, the Sato-Tate group $\operatorname{ST}(A)$ can be identified heuristically by computing the moments of $a_{p}$ and $a_{p^{2}}$ to sufficiently high precision and

\begin{tabular}{|c|c|c|c|}
\hline Type & $\operatorname{ST}(A)^{0}$ & $\overline{\operatorname{End}\left(A_{K}\right)_{\mathbb{R}}}$ & $A_{K}$ \\
\hline A & $\mathrm{USp}(4)$ & $\mathbb{R}$ & Generic abelian surface \\
\hline B & $\mathrm{SU}(2) \times \mathrm{SU}(2)$ & $\mathbb{R} \times \mathbb{R}$ & $\begin{array}{l}\mathrm{RM} \text { abelian surface or } \\
\text { product of non-isogenous generic elliptic curves }\end{array}$ \\
\hline $\mathbf{C}$ & $\mathrm{U}(1) \times \mathrm{SU}(2)$ & $\mathbb{C} \times \mathbb{R}$ & Product of generic elliptic curve and CM elliptic curve \\
\hline $\mathbf{D}$ & $\mathrm{U}(1) \times \mathrm{U}(1)$ & $\mathbb{C} \times \mathbb{C}$ & $\begin{array}{l}\mathrm{CM} \text { abelian surface or } \\
\text { product of non-isogenous CM elliptic curves }\end{array}$ \\
\hline $\mathbf{E}$ & $\mathrm{SU}(2)$ & $\mathrm{M}_{2}(\mathbb{R})$ & $\begin{array}{l}\text { QM abelian surface or } \\
\text { square of a generic elliptic curve }\end{array}$ \\
\hline $\mathbf{F}$ & $\mathrm{U}(1)$ & $\mathrm{M}_{2}(\mathbb{C})$ & Square of an elliptic curve with CM \\
\hline
\end{tabular}

TABLE 1. Sato-Tate identity components and real endomorphism algebras of abelian surfaces. 
consulting the corresponding tables [18, Tables 9-10]. To obtain a rigorous identification that does not depend on the Sato-Tate conjecture, one computes the Galois module $\operatorname{End}\left(A_{K}\right)_{\mathbb{R}}$ (with the natural action of $\operatorname{Gal}(K / F)$ ) and applies the explicit correspondence with Sato-Tate groups [18, Theorem 4.3].

\section{Conductor and bad Euler factors}

In this section we describe the computation of the conductor $N \in \mathbb{Z}$ of an abelian surface $A / \mathbb{Q}$ that is the Jacobian of a genus-2 curve $X / \mathbb{Q}$ with minimal discriminant $\Delta_{\min }$; for further background and the definition of the conductor, see Brumer and Kramer [7].

\subsection{Computing the conductor}

Only primes dividing $\Delta_{\min }$ can divide the conductor $N$ (but the converse need not hold), as shown by Liu [31]. For an odd prime $p \mid \Delta_{\text {min }}$, the exponent $\operatorname{ord}_{p}(N)$ and the $L$-factor $L_{p}(T)$ can be computed using the algorithm of Liu [32] computing the stable reduction of the curve, implemented in Pari by Liu and Henri Cohen and in Magma by Tim and Vladimir Dokchitser. There is also further recent work of Bouw and Wewers [9] computing these invariants using the semistable model instead, and in theory this method will also compute $\operatorname{ord}_{2}(N)$ (the 'wild' case). However, a general implementation of this method is not yet available.

\subsection{Analytically computing the conductor}

So it remains to compute $\operatorname{ord}_{2}(N)$, and we do so analytically, using methods that go back at least to Dokchitser [15] and the first author [4]; see also recent work of Farmer et al. [17]. The result of our computation will be that if the genus- 2 curve has an $L$-function with analytic continuation and functional equation, then there is provably only one possibility for the conductor and bad $L$-factor. One could take this unique possibility as the definition of $\operatorname{ord}_{2}(N)$ and the $L$-factor, and it must agree with the standard definition if the Hasse-Weil conjecture is true. Moreover, by multiplicity 1 for the Selberg class [43], there is never more than one permissible choice of the bad $L$-factor, so a suitably general version of the method will always succeed.

Let $A$ be an abelian surface over $\mathbb{Q}$ and let

$$
L(A, s)=\prod_{p} L_{p}\left(A, p^{-s}\right)^{-1}=\sum_{n=1}^{\infty} a_{n} n^{-s}
$$

be its Hasse-Weil $L$-function. Let $\Lambda(A, s)=\Gamma_{\mathbb{C}}(s)^{2} L(A, s)$, where $\Gamma_{\mathbb{C}}(s)=2(2 \pi)^{-s} \Gamma(s)$. Then conjecturally $\Lambda(A, s)$ continues to an entire function of order 1 and satisfies the functional equation

$$
\Lambda(A, s)=w N^{1-s} \Lambda(A, 2-s),
$$

where $N \in \mathbb{Z}_{\geqslant 1}$ is the conductor of $A$ and $w= \pm 1$ is its root number.

For $x>0$ we define

$$
S(x)=\frac{1}{x} \sum_{n=1}^{\infty} a_{n} K_{0}(4 \pi \sqrt{n / x}),
$$

where $K_{0}(y)=\int_{0}^{\infty} e^{-y \cosh t} d t$ is the $K$-Bessel function. Recall that $K_{0}(y) \sim \sqrt{(\pi / 2 y)} e^{-y}$ as $y \rightarrow \infty$ and $K_{0}(y) \sim \log (1 / y)$ as $y \rightarrow 0^{+}$; thus, $S(x)$ decays rapidly as $x \rightarrow 0^{+}$, and from the estimate $a_{n} \ll n^{1 / 2+\varepsilon}$ we have $S(x) \ll x^{1 / 2+\varepsilon}$ for large $x$. Therefore, the integral

$$
I(s)=\int_{0}^{\infty} S(x) x^{-s} d x
$$


is absolutely convergent for $\Re(s)>3 / 2$. For $s$ in that region we may also change the order of sum and integral and make the substitution $x \mapsto(4 \pi / y)^{2} n$ to obtain

$$
\begin{aligned}
I(s) & =\sum_{n=1}^{\infty} a_{n} \int_{0}^{\infty} K_{0}(4 \pi \sqrt{n / x}) x^{-s} \frac{d x}{x}=\sum_{n=1}^{\infty} a_{n} \int_{0}^{\infty} 2\left((4 \pi / y)^{2} n\right)^{-s} K_{0}(y) \frac{d y}{y} \\
& =2(4 \pi)^{-2 s} L(A, s) \int_{0}^{\infty} K_{0}(y) y^{2 s-1} d y .
\end{aligned}
$$

Finally, from the Mellin transform identity $\int_{0}^{\infty} K_{0}(y) y^{s-1} d y=2^{s-2} \Gamma(s / 2)^{2}$, we arrive at

$$
I(s)=\frac{1}{8} \Lambda(A, s) \text { for } \operatorname{Re}(s)>\frac{3}{2} .
$$

By a classical argument of Hecke, it then follows that the conjectured analytic continuation, growth properties and functional equation (5.2.1) of $\Lambda(A, s)$ are equivalent to the identity

$$
S(x)=w S(N / x) \text { for all } x>0 .
$$

Given a purported sequence of Dirichlet coefficients $\left\{a_{n}\right\}$ and values for $N$ and $w$, this gives a falsifiable identity that we can use to test for consistency with the Hasse-Weil conjecture. To carry this out in practice, we fix a suitable constant $C>0$ and consider the truncated sum

$$
S_{C}(x)=\frac{1}{x} \sum_{n \leqslant C x} a_{n} K_{0}(4 \pi \sqrt{n / x}) .
$$

Using the Ramanujan bound for $a_{n}$, one obtains the following estimate for the truncation error, provided that $C \geqslant 5$ :

$$
\left|S(x)-S_{C}(x)\right|<4 C x^{1 / 4}(1+2 x \sqrt{C}) e^{-4 \pi \sqrt{C}} .
$$

Also, since we might not know the values of $a_{n}$ for even $n$, we use the fact that $L(A, s)$ is given by an Euler product to write

$$
S_{C}(x)=\frac{1}{x} \sum_{j=0}^{\infty} a_{2^{j}} \sum_{\substack{m \leqslant C x 2^{-j} \\ 2 \nmid m}} a_{m} K_{0}\left(4 \pi \sqrt{m 2^{j} / x}\right)=\sum_{j=0}^{\left\lfloor\log _{2}(C x)\right\rfloor} \frac{a_{2^{j}}}{2^{j}} S_{C}^{\text {odd }}\left(\frac{x}{2^{j}}\right)
$$

where

$$
S_{C}^{\text {odd }}(x)=\frac{1}{x} \sum_{\substack{n \leqslant C x \\ 2 \nmid n}} a_{n} K_{0}(4 \pi \sqrt{n / x}) .
$$

We then simply try all possibilities for $N, w$, and the Euler factor $L_{2}(A, T)$ to see which, if any, give answers consistent with (5.2.2). Specifically, we compute $S\left(2^{1 / 4} \sqrt{N}\right)-w S\left(2^{-1 / 4} \sqrt{N}\right)$ for each candidate set of parameters and check whether any of these are zero. By Brumer and Kramer [7, Theorem 6.2] we have $\operatorname{ord}_{2}(N) \leqslant 20$. Moreover, in the case where $A$ is the Jacobian of a genus-2 curve of discriminant $\Delta_{\min }$, by Liu [31] we have $N \mid \Delta_{\min }$, so we may assume that $\operatorname{ord}_{2}(N) \leqslant \min \left(20, \operatorname{ord}_{2}\left(\Delta_{\text {min }}\right)\right)$. Thus, by (5.2.3) and (5.2.4), our computation reduces to that of $S_{C}^{\text {odd }}\left(\sqrt{2^{k-1 / 2} N_{\text {odd }}}\right)$ for each $k$ with $0 \leqslant k \leqslant \min \left(20, \operatorname{ord}_{2}\left(\Delta_{\text {min }}\right)\right)+1$, where $N_{\text {odd }}$ denotes the odd part of the conductor.

\subsection{Results}

We coded the above procedure in interval arithmetic, based on Fredrik Johansson's library Arb [28], using 53 bits of internal precision and $C=10$. In every case that we tested, we found exactly one consistent choice of $N, w$, and $L_{2}(A, T)$. 
Example 5.3.1. Consider the genus-2 curve

$$
y^{2}+\left(x^{3}+x+1\right) y=-x^{6}+6 x^{4}+10 x^{3}-33 x^{2}-14 x+3
$$

of minimal discriminant $\Delta_{\text {min }}=477696=2^{9} \cdot 3 \cdot 311$. Running through all positive integers $N$ with $3 \cdot 311|N| \Delta_{\min }, w= \pm 1$, and all choices of $L_{2}(A, T)$, we find that for $N=3732=$ $2^{2} \cdot 3 \cdot 311, w=1$, and $L_{2}(A, T)=1-T+T^{2}$, the value of $S\left(2^{1 / 4} \sqrt{N}\right)-w S\left(2^{-1 / 4} \sqrt{N}\right)$ lies in an interval with centre very near 0 and radius less than $1.24 \times 10^{-11}$. (Most of the uncertainty in this value comes from the error term (5.2.3).) For every other choice of $N, w$ or $L_{2}(A, T)$, we find, provably, that $\left|S\left(2^{1 / 4} \sqrt{N}\right)-w S\left(2^{-1 / 4} \sqrt{N}\right)\right|>3.3 \times 10^{-7}$.

REMARK 5.3.2. One can treat all of the $a_{n}$ as unknowns (not only the ones for even $n$ ), subject only to the assumptions that they are integer-valued and satisfy the Ramanujan bound. This was carried out by Farmer et al. [17], who found all solutions to (5.2.1) with $N \leqslant 500$. Assuming the Hasse-Weil conjecture, this includes a complete classification, up to isogeny, of the abelian surfaces with conductor 500 or less.

REMARK 5.3.3. It is possible for a genus- 2 curve to have bad reduction at a prime $p$ that does not divide the conductor, so $p \mid \Delta_{\min }$ but $p \nmid N$. When this occurs $\Delta_{\min }$ is typically divisible by a large power of $p$ (typically $p^{12}$ ), and consequently much larger than $10^{6}$; it is thus not surprising that none of the curves in our database have bad reduction at a prime that does not divide the conductor.

\section{Endomorphism data}

Let $X: y^{2}=f(x)$ be a simplified Weierstrass model of a genus-2 curve over $\mathbb{Q}$, and let $A:=\operatorname{Jac}(X)$ be its Jacobian. The endomorphism ring $\operatorname{End}(A)$ is an important invariant of the curve (recall from $\S 2$ that the elements of $\operatorname{End}(A)$ are defined over $\mathbb{Q}$ ): for example, the presence of non-trivial idempotents in $\operatorname{End}(A)$ indicates that $A$ splits as a product of elliptic curves. More coarsely, the $\mathbb{Q}$-algebra $\operatorname{End}(A)_{\mathbb{Q}}$ contains information about the isogeny class of $A$, and the presence of non-trivial idempotents in this algebra indicates whether $A$ is $\mathbb{Q}$-isogenous to a product of elliptic curves. Finally, if $K$ is the minimal field over which all the endomorphisms of $A$ are defined, then as mentioned in $\S 4.4$, the $\mathbb{R}$-algebra $\operatorname{End}\left(A_{K}\right)_{\mathbb{R}}$ determines and is determined by the identity component $\mathrm{ST}^{0}(A)$ of the Sato-Tate group of $A$.

Our approach to determining $\operatorname{End}(A)$ is to first determine $\operatorname{End}\left(A_{\overline{\mathbb{Q}}}\right)$, and thereby $\operatorname{End}\left(A_{\overline{\mathbb{Q}}}\right)_{\mathbb{Q}}$ and $\operatorname{End}\left(A_{\overline{\mathbb{Q}}}\right)_{\mathbb{R}}$. We have $\operatorname{End}\left(A_{\overline{\mathbb{Q}}}\right)=\operatorname{End}\left(A_{\mathbb{C}}\right)$, so we employ transcendental methods to determine these rings. (Consequently, our results are not fully rigorous, as they could in principle depend on the precision of our calculations; this is something we hope to rectify in future work.) From this, we obtain a candidate for the minimal number field $K$ over which all endomorphisms of $A$ are defined, and for each subfield $F \subset K$ we can then $\operatorname{compute} \operatorname{End}\left(A_{F}\right)$ by taking Galois invariants. In other words, we can determine the structure of $\operatorname{End}\left(A_{\overline{\mathbb{Q}}}\right)$ as a $\operatorname{Gal}(\overline{\mathbb{Q}} / \mathbb{Q})$-module.

\subsection{Reduction to linear algebra}

We apply the techniques of van Wamelen [47]. The Abel-Jacobi map gives a holomorphic isomorphism $A(\mathbb{C}) \simeq V / \Lambda$, where $V=H^{0}\left(X, \omega_{X}\right)^{\vee}$ is the dual vector space of the global differentials of our genus $g=2$ curve $X$, identified with the tangent space of $A$ at 0 , and $\Lambda \subset V$ is given by $H_{1}(X, \mathbb{Z})$ (via the embedding coming from integration of forms along paths). Concretely, after choosing bases, $\Lambda$ can be embedded as a lattice in $\mathbb{C}^{g}$ using the periods of $X$, that is, the integrals $\int_{\delta_{j}} \omega_{i}$, where $\omega_{1}, \ldots, \omega_{g}$ is a basis for $H^{0}\left(X, \omega_{X}\right)$, and $\delta_{1}, \ldots, \delta_{2 g}$ is a 
basis for $H_{1}(X, \mathbb{Z})$. These periods can be evaluated by numerical methods; an especially fast technique when $g=2$ uses the arithmetic-geometric mean and is due to Dupont [16].

We now find the endomorphisms of this abelian variety using the LLL algorithm [30]. To do this, we consider the lattice $\Lambda$ not as a subset of $\mathbb{C}^{g}$, but rather as the standard lattice $\mathbb{Z}^{2 g} \subset \mathbb{R}^{2 g}$ equipped with a complex structure, that is, a map $J: \mathbb{R}^{2 g} \rightarrow \mathbb{R}^{2 g}$ such that $J^{2}=-1$. The elements of $\operatorname{End}\left(A_{\overline{\mathbb{Q}}}\right)$ can then be identified with the maps $R: \mathbb{Z}^{2 g} \rightarrow \mathbb{Z}^{2 g}$ that satisfy

$$
R J=J R
$$

Equation (6.1.1) is linear in the integer entries of $R$, and given an approximation of $A$, a basis of approximate solutions of this equation can be found using the LLL algorithm. In practice, we apply this algorithm after determining the period matrix of $X$ (and with it, the matrix $J$ ) up to 200 digits of precision. Having in this way found a basis $R_{1}, \ldots, R_{d}$ of the ring $\operatorname{End}\left(A_{\overline{\mathbb{Q}}}\right)$ in terms of matrices $R_{i}: \mathbb{Z}^{2 g} \rightarrow \mathbb{Z}^{2 g}$, we then apply standard meataxe algorithms [26] to determine the algebras $\operatorname{End}\left(A_{\overline{\mathbb{Q}}}\right)_{\mathbb{Q}}$. We may then describe the $\operatorname{ring} \operatorname{End}\left(A_{\overline{\mathbb{Q}}}\right)$ as an order in $\operatorname{End}\left(A_{\overline{\mathbb{Q}}}\right)_{\mathbb{Q}}$.

As a sanity check, we verify that (to high precision) the structure of the real endomorphism algebra $\operatorname{End}\left(A_{\overline{\mathbb{Q}}}\right)_{\mathbb{R}}$ agrees with that implied by the Sato-Tate group of $X$, which we (heuristically) computed as described in $\S 4.4$ using entirely different methods.

\subsection{Galois module structure}

The ring $\operatorname{End}\left(A_{\overline{\mathbb{Q}}}\right)$ is a $\operatorname{Gal}(\overline{\mathbb{Q}} / \mathbb{Q})$-module in a natural way, and $\operatorname{End}(A)$ can be recovered by taking Galois invariants, that is, by determining the elements of $\operatorname{End}\left(A_{\overline{\mathbb{Q}}}\right)$ that are fixed under the Galois action. This reduces the question to linear algebra over $\mathbb{Q}$.

As our curve $X$ is defined over $\mathbb{Q}$, the tangent space of $A_{\mathbb{Q}}$ at 0 comes equipped with a natural action of $\operatorname{Gal}(\overline{\mathbb{Q}} / \mathbb{Q})$. The space $H^{0}\left(X_{\overline{\mathbb{Q}}}, \omega_{X_{\overline{\mathbb{Q}}}}\right)$ that is dual to this tangent space has a basis $d x / y, x d x / y$ defined over $\mathbb{Q}$. We use this to determine the Galois action on $\operatorname{End}\left(A_{\overline{\mathbb{Q}}}\right)$; denoting the period matrix of $A$ with respect to the given basis of differentials by $\Pi$, the equality

$$
M \Pi=\Pi R
$$

allows us to convert any basis element $R_{i}: \mathbb{Z}^{4} \rightarrow \mathbb{Z}^{4}$ above into a matrix $M_{i}: \mathbb{C}^{2} \rightarrow \mathbb{C}^{2}$. By the argument above, the entries of the matrices $M_{i}$ lie in $\overline{\mathbb{Q}}$, and $\operatorname{Gal}(\overline{\mathbb{Q}} / \mathbb{Q})$ acts on $M_{i}$ entrywise. Using LLL once more, we can determine a putative Galois number field $L$ over which all these entries are defined. And given a subfield $K$ of $L$, we can determine $\operatorname{End}\left(A_{K}\right)$ by solving a system of linear equations: we find combinations $\sum_{i=1}^{d} n_{i} M_{i}$ that are stable under all generators $\sigma$ of the corresponding Galois subgroup, that is, rational solutions $n_{i}$ to the system of equations

$$
\sum_{i=1}^{d} n_{i}\left(M_{i}^{\sigma}-M_{i}\right)=0,
$$

with $\sigma$ running over the aforementioned generators. To determine the endomorphism ring over $K$, we in fact need a basis of the integral solutions of (6.2.2), but this can be efficiently recovered from the basis over $\mathbb{Q}$ using lattice saturation techniques.

\subsection{Decomposition up to isogeny}

The situation where $\operatorname{End}\left(A_{\overline{\mathbb{Q}}}\right)_{\mathbb{Q}}$ contains a non-trivial idempotent $e$ is of particular interest. We show in this section how to obtain a splitting of $A \stackrel{\sim}{\rightarrow} E \times E^{\prime}$ into a product of two elliptic curves, up to isogeny. 
The idempotent $e$ gives rise to a matrix $M_{e}: \mathbb{C}^{2} \rightarrow \mathbb{C}^{2}$ whose image has dimension 1 . In practice, this means that we restrict to a single factor of $\mathbb{C}^{2}$, one in which the image of the lattice $\Lambda$ under $M_{e}$ does not become trivial. We thus obtain an isogeny

$$
L_{e}: \mathbb{C}^{2} / \Lambda \rightarrow \mathbb{C} / \Lambda_{e}
$$

where $\Lambda_{e}$ is a lattice in $\mathbb{C}$. Using the values $g_{4}\left(\Lambda_{e}\right), g_{6}\left(\Lambda_{e}\right)$ of the classical elliptic functions, we obtain an elliptic curve $E$ whose complex points are given by $\mathbb{C} / \Lambda_{e}$. What is more, the properties of these functions together with our $\mathbb{Q}$-rational choice of basis of $H^{0}\left(X_{\mathbb{C}}, \omega_{X_{\mathbb{C}}}\right)$ ensure that the map $L_{e}$ is defined over a field that is as small as possible. Indeed, in all cases where $e$ was defined over $\mathbb{Q}$ we have obtained rational elliptic curves $E$ whose primes of bad reduction are a subset of those of $X$.

An online implementation of the methods discussed in this section in a mixture of MAGMA [6], PARi [38] and SageMath [40] can be found at [41]. We conclude by giving some examples.

\subsection{Examples}

EXAMPLE 6.4.1. We first consider the curve

$$
X: y^{2}+\left(x^{2}+x\right) y=x^{5}+x^{4}+2 x^{3}+x^{2}+x .
$$

An approximation of the period matrix for this curve with respect to the basis $\{d x / y, x d x / y\}$ is given by

$$
\left(\begin{array}{cccc}
3.938+1.418 i & 0.072+9.184 i & -1.933-2.650 i & 1.933-2.650 i \\
-2.005-6.534 i & -5.871-4.069 i & -1.933+2.650 i & 1.933+2.650 i
\end{array}\right) .
$$

Using the LLL methods sketched above, we find that with respect to the chosen homology basis, the endomorphism ring is generated as a $\mathbb{Z}$-module by the three elements

$$
\left(\begin{array}{llll}
1 & 0 & 0 & 0 \\
0 & 1 & 0 & 1 \\
0 & 0 & 1 & 0 \\
0 & 0 & 0 & 1
\end{array}\right), \quad\left(\begin{array}{cccc}
0 & 1 & 0 & 1 \\
1 & 0 & -1 & 0 \\
0 & 0 & 0 & 1 \\
0 & 0 & 1 & 0
\end{array}\right), \quad\left(\begin{array}{cccc}
-1 & 1 & -2 & 3 \\
2 & -2 & 2 & -4 \\
1 & -1 & 0 & -1 \\
-1 & 1 & 0 & 1
\end{array}\right)
$$

Via equality (6.2.1), these correspond to the complex matrices

$$
\left(\begin{array}{cc}
1.000 & 0.000 \\
0.000 & 1.000
\end{array}\right), \quad\left(\begin{array}{cc}
0.000 & -1.000 \\
-1.000 & 0.000
\end{array}\right), \quad\left(\begin{array}{cc}
-0.500+1.323 i & -0.500+1.323 i \\
-0.500+1.323 i & -0.500+1.323 i
\end{array}\right)
$$

which are approximations of the following matrices over $\mathbb{Q}(\sqrt{-7})$ :

$$
\left(\begin{array}{ll}
1 & 0 \\
0 & 1
\end{array}\right), \quad\left(\begin{array}{cc}
0 & -1 \\
-1 & 0
\end{array}\right), \quad\left(\begin{array}{cc}
(-1+\sqrt{-7}) / 2 & (-1+\sqrt{-7}) / 2 \\
(-1+\sqrt{-7}) / 2 & (-1+\sqrt{-7}) / 2
\end{array}\right)
$$

The solutions to the equations (6.2.2) are easy to spot in this case: the elements of $\operatorname{End}(A)$ are exactly those in the span of the first two matrices.

It follows that $\operatorname{End}(A)_{\mathbb{Q}}$ is isomorphic to $\mathbb{Q} \times \mathbb{Q}$; in this algebra, $\operatorname{End}(A)$ corresponds to an order of index 2 in $\mathbb{Z} \times \mathbb{Z}$. A more careful study shows that $\operatorname{End}\left(A_{\overline{\mathbb{Q}}}\right)_{\mathbb{Q}} \simeq \mathbb{Q} \times \mathbb{Q}(\sqrt{-7})$, and $\operatorname{End}\left(A_{\overline{\mathbb{Q}}}\right)$ is an order of index 4 in $\mathbb{Z} \times \mathbb{Z}[(1+\sqrt{-7}) / 2]$.

We see that the Jacobian $A$ is already split over $\mathbb{Q}$. Constructing idempotents, we obtain 2 -isogenies to the rational elliptic curves with LMFDB labels 14.a5 and 49.a4. This agrees with the fact that the conductor of $X$ equals $686=2 \cdot 7^{3}$.

EXAMPLE 6.4.2. Our database contains only two curves with quaternionic multiplication $(\mathrm{QM})$, both of conductor $262144=2^{18}$, with equations $y^{2}=x^{5}-x^{4}-4 x^{3}-8 x^{2}+5 x-1$ and $y^{2}=x^{5}+x^{4}+4 x^{3}+8 x^{2}+5 x+1$; their Jacobians are isogenous quadratic twists and both admit QM by a maximal order in the rational quaternion algebra of discriminant 6 . 


\section{Appendix. Tables}

REMARK A.1. The fact that the average analytic rank in our database is significantly greater than the expected average value of 0.5 is likely due to the fact that having small coefficients made it more likely that $4 f+h^{2}$ will be a sextic whose leading coefficient is a square, in which case the difference of the two points at infinity is a rational divisor that typically has infinite order (it is torsion if and only if the continued fraction expansion of $y$ in the hyperelliptic function field $\mathbb{Q}\left[x, y\left[/\left(y^{2}-4 f-h^{2}\right)\right.\right.$ is periodic [2]). When this occurs the Mordell-Weil rank of the Jacobian is 1 greater than it would be generically.

TABLE A.1. Searching in boxes for genus-2 curves $y^{2}+h(x) y=f(x)$ with $|\Delta(f, h)| \leqslant 10^{n}$.

\begin{tabular}{lcrrrr}
\hline & & \multicolumn{4}{c}{ Discriminant bound } \\
\cline { 3 - 6 } Size & Box & $10^{3}$ & \multicolumn{1}{c}{$10^{4}$} & $10^{5}$ & \multicolumn{1}{c}{$10^{6}$} \\
\hline$\approx 10^{11}$ & $S_{1}(12)$ & 47 & 921 & 8301 & 56724 \\
$\approx 10^{12}$ & $S_{1}(17)$ & 50 & 953 & 8622 & 59482 \\
$\approx 10^{13}$ & $S_{1}(24)$ & 52 & 979 & 8852 & 61401 \\
$\approx 10^{14}$ & $S_{1}(33)$ & 53 & 987 & 8993 & 62696 \\
$\approx 10^{15}$ & $S_{1}(46)$ & 54 & 1007 & 9121 & 63697 \\
$\approx 10^{16}$ & $S_{1}(64)$ & 54 & 1020 & 9232 & 64524 \\
$\approx 10^{17}$ & $S_{1}(90)$ & 54 & 1028 & 9304 & 65093 \\
$\approx 10^{11}$ & $S_{2}(2,1.83)$ & 50 & 944 & 8486 & 58127 \\
$\approx 10^{12}$ & $S_{2}(2,2.02)$ & 51 & 967 & 8761 & 60546 \\
$\approx 10^{13}$ & $S_{2}(2,2.27)$ & 52 & 986 & 8921 & 62061 \\
$\approx 10^{14}$ & $S_{2}(2,2.53)$ & 52 & 1000 & 9053 & 63150 \\
$\approx 10^{15}$ & $S_{2}(2,2.83)$ & 54 & 1015 & 9150 & 64035 \\
$\approx 10^{16}$ & $S_{2}(2,3.16)$ & 54 & 1023 & 9217 & 64537 \\
$\approx 10^{17}$ & $S_{2}(2,3.51)$ & 56 & 1027 & 9274 & 64923 \\
$\approx 10^{11}$ & $S_{3}(3.00)$ & 48 & 937 & 8404 & 57402 \\
$\approx 10^{12}$ & $S_{3}(3.47)$ & 49 & 958 & 8590 & 59043 \\
$\approx 10^{13}$ & $S_{3}(4.00)$ & 50 & 978 & 8801 & 60859 \\
$\approx 10^{14}$ & $S_{3}(4.69)$ & 50 & 982 & 8870 & 61644 \\
$\approx 10^{15}$ & $S_{3}(5.37)$ & 52 & 999 & 9003 & 62740 \\
$\approx 10^{16}$ & $S_{3}(6.17)$ & 54 & 1007 & 9106 & 63543 \\
$\approx 10^{17}$ & $S_{3}(7.14)$ & 54 & 1010 & 9167 & 64021 \\
\hline & & & & &
\end{tabular}

TABLE A.2. Searching for integral models with discriminants $|\Delta(f, h)| \leqslant 10^{6}$.

\begin{tabular}{lcccc}
\hline Set & Cardinality & Models & Curves & Discs \\
\hline$S_{1}$ & $1.02 \times 10^{17}$ & 1908760 & 65093 & 56132 \\
$S_{2}$ & $9.84 \times 10^{16}$ & 1295050 & 64923 & 56009 \\
$S_{1} \cup S_{2}$ & $2.00 \times 10^{17}$ & 2147485 & 65638 & 56549 \\
$S_{3}$ & $1.01 \times 10^{17}$ & 1299092 & 64021 & 55240 \\
$S_{1} \cup S_{2} \cup S_{3}$ & $3.01 \times 10^{17}$ & 2218870 & 65892 & 56728 \\
$S_{4}$ & $2.10 \times 10^{16}$ & 1453127 & 64768 & 55820 \\
$S_{1} \cup S_{2} \cup S_{3} \cup S_{4}$ & $3.22 \times 10^{17}$ & 2232548 & 66130 & 56907 \\
\hline
\end{tabular}




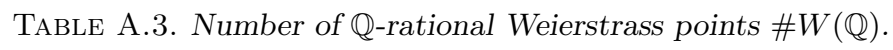

\begin{tabular}{crrrr}
\hline$\# W(\mathbb{Q})$ & Total & $\mathrm{GL}_{2}$-type & $\mathbb{Q}$-simple & $\overline{\mathbb{Q}}$-simple \\
\hline 0 & 32616 & 2265 & 30462 & 30326 \\
1 & 24611 & 46 & 24580 & 24574 \\
2 & 8005 & 490 & 7522 & 7461 \\
3 & 886 & 35 & 881 & 851 \\
4 & 40 & 10 & 32 & 20 \\
& 66158 & 2846 & 63477 & 63232 \\
\hline
\end{tabular}

TABLE A.4. Torsion subgroups.

\begin{tabular}{rcrrrr}
\hline$\# G$ & $G$ & Total & GL $_{2}$-type & $\mathbb{Q}$-simple & $\overline{\mathbb{Q}}$-simple \\
\hline 1 & $\{0\}$ & 44190 & 586 & 43694 & 43664 \\
2 & $\mathbb{Z} / 2 \mathbb{Z}$ & 14681 & 848 & 13845 & 13737 \\
3 & $\mathbb{Z} / 3 \mathbb{Z}$ & 2295 & 279 & 2019 & 2006 \\
4 & $\mathbb{Z} / 2 \mathbb{Z} \times \mathbb{Z} / 2 \mathbb{Z}$ & 1352 & 244 & 1143 & 1085 \\
4 & $\mathbb{Z} / 4 \mathbb{Z}$ & 1402 & 338 & 1065 & 1063 \\
5 & $\mathbb{Z} / 5 \mathbb{Z}$ & 725 & 84 & 650 & 650 \\
6 & $\mathbb{Z} / 6 \mathbb{Z}$ & 595 & 210 & 387 & 378 \\
7 & $\mathbb{Z} / 7 \mathbb{Z}$ & 97 & 13 & 86 & 86 \\
8 & $\mathbb{Z} / 8 \mathbb{Z}$ & 201 & 28 & 175 & 174 \\
8 & $\mathbb{Z} / 2 \mathbb{Z} \times \mathbb{Z} / 4 \mathbb{Z}$ & 159 & 92 & 67 & 66 \\
8 & $\mathbb{Z} / 2 \mathbb{Z} \times \mathbb{Z} / 2 \mathbb{Z} \times \mathbb{Z} / 2 \mathbb{Z}$ & 33 & 5 & 30 & 18 \\
9 & $\mathbb{Z} / 9 \mathbb{Z}$ & 30 & 1 & 30 & 29 \\
9 & $\mathbb{Z} / 3 \mathbb{Z} \times \mathbb{Z} / 3 \mathbb{Z}$ & 8 & 5 & 2 & 0 \\
10 & $\mathbb{Z} / 10 \mathbb{Z}$ & 131 & 5 & 127 & 126 \\
11 & $\mathbb{Z} / 11 \mathbb{Z}$ & 8 & 2 & 8 & 8 \\
12 & $\mathbb{Z} / 12 \mathbb{Z}$ & 59 & 40 & 19 & 18 \\
12 & $\mathbb{Z} / 2 \mathbb{Z} \times \mathbb{Z} / 6 \mathbb{Z}$ & 66 & 22 & 47 & 44 \\
13 & $\mathbb{Z} / 13 \mathbb{Z}$ & 7 & 0 & 7 & 7 \\
14 & $\mathbb{Z} / 14 \mathbb{Z}$ & 12 & 1 & 12 & 12 \\
15 & $\mathbb{Z} / 15 \mathbb{Z}$ & 17 & 7 & 10 & 10 \\
16 & $\mathbb{Z} / 16 \mathbb{Z}$ & 3 & 0 & 3 & 3 \\
16 & $\mathbb{Z} / 2 \mathbb{Z} \times \mathbb{Z} / 8 \mathbb{Z}$ & 31 & 11 & 20 & 20 \\
16 & $\mathbb{Z} / 4 \mathbb{Z} \times \mathbb{Z} / 4 \mathbb{Z}$ & 3 & 2 & 0 & 0 \\
16 & $\mathbb{Z} / 2 \mathbb{Z} \times \mathbb{Z} / 2 \mathbb{Z} \times \mathbb{Z} / 4 \mathbb{Z}$ & 3 & 2 & 1 & 1 \\
17 & $\mathbb{Z} / 17 \mathbb{Z}$ & 1 & 0 & 1 & 1 \\
18 & $\mathbb{Z} / 18 \mathbb{Z}$ & 3 & 0 & 3 & 3 \\
18 & $\mathbb{Z} / 3 \mathbb{Z} \times \mathbb{Z} / 6 \mathbb{Z}$ & 6 & 5 & 0 & 0 \\
19 & $\mathbb{Z} / 19 \mathbb{Z}$ & 1 & 1 & 1 & 0 \\
\hline & & & & &
\end{tabular}


Table A.4 (Continued).

\begin{tabular}{ccrrrr}
\hline$\# G$ & $G$ & Total & GL $_{2}$-type & $\mathbb{Q}$-simple & $\overline{\mathbb{Q}}$-simple \\
\hline 20 & $\mathbb{Z} / 20 \mathbb{Z}$ & 6 & 1 & 5 & 5 \\
20 & $\mathbb{Z} / 2 \mathbb{Z} \times \mathbb{Z} / 10 \mathbb{Z}$ & 7 & 2 & 6 & 5 \\
21 & $\mathbb{Z} / 21 \mathbb{Z}$ & 5 & 3 & 3 & 2 \\
22 & $\mathbb{Z} / 22 \mathbb{Z}$ & 2 & 0 & 2 & 2 \\
24 & $\mathbb{Z} / 24 \mathbb{Z}$ & 4 & 3 & 1 & 1 \\
24 & $\mathbb{Z} / 2 \mathbb{Z} \times \mathbb{Z} / 12 \mathbb{Z}$ & 4 & 2 & 2 & 2 \\
24 & $\mathbb{Z} / 2 \mathbb{Z} \times \mathbb{Z} / 2 \mathbb{Z} \times \mathbb{Z} / 6 \mathbb{Z}$ & 2 & 1 & 1 & 1 \\
27 & $\mathbb{Z} / 27 \mathbb{Z}$ & 1 & 0 & 1 & 1 \\
27 & $\mathbb{Z} / 3 \mathbb{Z} \times \mathbb{Z} / 9 \mathbb{Z}$ & 1 & 1 & 0 & 0 \\
28 & $\mathbb{Z} / 28 \mathbb{Z}$ & 1 & 0 & 1 & 1 \\
28 & $\mathbb{Z} / 2 \mathbb{Z} \times \mathbb{Z} / 14 \mathbb{Z}$ & 1 & 0 & 1 & 1 \\
29 & $\mathbb{Z} / 29 \mathbb{Z}$ & 1 & 0 & 1 & 1 \\
32 & $\mathbb{Z} / 2 \mathbb{Z} \times \mathbb{Z} / 2 \mathbb{Z} \times \mathbb{Z} / 8 \mathbb{Z}$ & 2 & 2 & 0 & 0 \\
36 & $\mathbb{Z} / 6 \mathbb{Z} \times \mathbb{Z} / 6 \mathbb{Z}$ & 1 & 0 & 0 & 0 \\
39 & $\mathbb{Z} / 39 \mathbb{Z}$ & 1 & 0 & 1 & 1 \\
& & 66158 & 2846 & 63477 & 63232 \\
\hline
\end{tabular}

TABle A.5. Automorphism groups.

\begin{tabular}{ccrrrr}
\hline Aut $(X)$ & Aut $\left(X_{\overline{\mathbb{Q}}}\right)$ & Total & $\mathrm{GL}_{2}$-type & $\mathbb{Q}$-simple & $\overline{\mathbb{Q}}$-simple \\
\hline$C_{2}$ & $C_{2}$ & 63310 & 174 & 63234 & 63227 \\
$C_{2}$ & $V_{4}$ & 125 & 1 & 125 & 0 \\
$C_{2}$ & $D_{8}$ & 17 & 0 & 17 & 0 \\
$C_{2}$ & $C_{10}$ & 5 & 0 & 5 & 5 \\
$C_{2}$ & $D_{12}$ & 23 & 1 & 23 & 0 \\
$C_{4}$ & $D_{8}$ & 13 & 13 & 13 & 0 \\
$C_{4}$ & $\tilde{S}_{4}$ & 2 & 2 & 2 & 0 \\
$V_{4}$ & $V_{4}$ & 2573 & 2573 & 0 & 0 \\
$V_{4}$ & $D_{8}$ & 16 & 16 & 0 & 0 \\
$V_{4}$ & $D_{12}$ & 3 & 3 & 0 & 0 \\
$V_{4}$ & $2 D_{12}$ & 4 & 4 & 0 & 0 \\
$V_{4}$ & $\tilde{S}_{4}$ & 1 & 1 & 0 & 0 \\
$C_{6}$ & $D_{12}$ & 58 & 58 & 58 & 0 \\
$D_{8}$ & $D_{8}$ & 4 & 0 & 0 & 0 \\
$D_{12}$ & $D_{12}$ & 4 & 0 & 0 & 0 \\
& & 66158 & 2846 & 63477 & 63232 \\
\hline
\end{tabular}


TABLE A.6. Sato-Tate groups.

\begin{tabular}{ccrrrr}
\hline Type & ST & Total & GL $_{2}$-type & Q-simple & $\overline{\mathbb{Q}}$-simple \\
\hline $\mathbf{A}$ & $\mathrm{USp}(4)$ & 63107 & 0 & 63107 & 63107 \\
$\mathbf{B}$ & $G_{3,3}$ & 2440 & 2440 & 97 & 97 \\
$\mathbf{B}$ & $N\left(G_{3,3}\right)$ & 144 & 0 & 144 & 19 \\
$\mathbf{C}$ & $N\left(G_{1,3}\right)$ & 303 & 303 & 0 & 0 \\
$\mathbf{D}$ & $F_{a c}$ & 6 & 0 & 6 & 6 \\
$\mathbf{D}$ & $F_{a, b}$ & 0 & 0 & 0 & 0 \\
$\mathbf{E}$ & $E_{1}$ & 8 & 0 & 0 & 0 \\
$\mathbf{E}$ & $E_{2}$ & 3 & 3 & 3 & 0 \\
$\mathbf{E}$ & $E_{3}$ & 7 & 7 & 7 & 0 \\
$\mathbf{E}$ & $E_{4}$ & 10 & 10 & 10 & 0 \\
$\mathbf{E}$ & $E_{6}$ & 51 & 51 & 51 & 0 \\
$\mathbf{E}$ & $J\left(E_{1}\right)$ & 24 & 24 & 2 & 0 \\
$\mathbf{E}$ & $J\left(E_{2}\right)$ & 9 & 0 & 9 & 2 \\
$\mathbf{E}$ & $J\left(E_{3}\right)$ & 4 & 0 & 4 & 0 \\
$\mathbf{E}$ & $J\left(E_{4}\right)$ & 17 & 0 & 17 & 1 \\
$\mathbf{E}$ & $J\left(E_{6}\right)$ & 17 & 0 & 17 & 0 \\
$\mathbf{F}$ & $J\left(C_{2}\right)$ & 2 & 2 & 2 & 0 \\
$\mathbf{F}$ & $J\left(C_{4}\right)$ & 1 & 1 & 0 & 0 \\
$\mathbf{F}$ & $J\left(C_{6}\right)$ & 0 & 0 & 0 & 0 \\
$\mathbf{F}$ & $J\left(D_{2}\right)$ & 0 & 0 & 0 & 0 \\
$\mathbf{F}$ & $J\left(D_{3}\right)$ & 0 & 0 & 0 & 0 \\
$\mathbf{F}$ & $J\left(D_{4}\right)$ & 0 & 0 & 0 & 0 \\
$\mathbf{F}$ & $J\left(D_{6}\right)$ & 0 & 0 & 0 & 0 \\
$\mathbf{F}$ & $J(T)$ & 0 & 0 & 0 & 0 \\
$\mathbf{F}$ & $J(O)$ & 0 & 0 & 0 & 0 \\
$\mathbf{F}$ & $C_{2,1}$ & 0 & 0 & 0 & 0 \\
$\mathbf{F}$ & $C_{6,1}$ & 0 & 0 & 0 & 0 \\
$\mathbf{F}$ & $D_{2,1}$ & 3 & 3 & 0 & 0 \\
$\mathbf{F}$ & $D_{4,1}$ & 0 & 0 & 0 & 0 \\
$\mathbf{F}$ & $D_{6,1}$ & 0 & 0 & 0 & 0 \\
$\mathbf{F}$ & $D_{3,2}$ & 1 & 1 & 0 & 0 \\
$\mathbf{F}$ & $D_{4,2}$ & 0 & 0 & 0 & 0 \\
$\mathbf{F}$ & $D_{6,2}$ & 1 & 1 & 0 & 0 \\
$\mathbf{F}$ & $O_{1}$ & 0 & 0 & 0 & 0 \\
\hline & & 66158 & 2846 & 63477 & 63232 \\
\hline $\mathbf{F}$ & & & & &
\end{tabular}


TABLE A.7. 2-Selmer rank, torsion rank, analytic rank, Ш 2-rank, and averages.

\begin{tabular}{|c|c|c|c|c|}
\hline 2-Selmer rank & $\operatorname{Jac}(X)[2] \mathrm{rank}$ & Analytic rank & $\amalg[2] \mathrm{rank}$ & Total \\
\hline 0 & 0 & 0 & 0 & 3878 \\
\hline 1 & 0 & 0 & 1 & 604 \\
\hline 1 & 0 & 1 & 0 & 20191 \\
\hline 1 & 1 & 0 & 0 & 4701 \\
\hline 2 & 0 & 0 & 2 & 445 \\
\hline 2 & 0 & 1 & 1 & 680 \\
\hline 2 & 0 & 2 & 0 & 18481 \\
\hline 2 & 1 & 0 & 1 & 477 \\
\hline 2 & 1 & 1 & 0 & 8434 \\
\hline 2 & 2 & 0 & 0 & 886 \\
\hline 3 & 0 & 0 & 3 & 4 \\
\hline 3 & 0 & 1 & 2 & 173 \\
\hline 3 & 0 & 2 & 1 & 88 \\
\hline 3 & 0 & 3 & 0 & 2832 \\
\hline 3 & 1 & 0 & 2 & 908 \\
\hline 3 & 1 & 1 & 1 & 307 \\
\hline 3 & 1 & 2 & 0 & 1958 \\
\hline 3 & 2 & 0 & 1 & 44 \\
\hline 3 & 2 & 1 & 0 & 529 \\
\hline 3 & 3 & 0 & 0 & 36 \\
\hline 4 & 0 & 2 & 2 & 2 \\
\hline 4 & 0 & 4 & 0 & 10 \\
\hline 4 & 1 & 0 & 3 & 24 \\
\hline 4 & 1 & 1 & 2 & 217 \\
\hline 4 & 1 & 2 & 1 & 16 \\
\hline 4 & 1 & 3 & 0 & 44 \\
\hline 4 & 2 & 0 & 2 & 101 \\
\hline 4 & 2 & 1 & 1 & 17 \\
\hline 4 & 2 & 2 & 0 & 13 \\
\hline 4 & 3 & 1 & 0 & 4 \\
\hline 5 & 1 & 0 & 4 & 9 \\
\hline 5 & 1 & 1 & 3 & 7 \\
\hline 5 & 1 & 2 & 2 & 1 \\
\hline 5 & 1 & 3 & 1 & 1 \\
\hline 5 & 2 & 0 & 3 & 12 \\
\hline 5 & 2 & 1 & 2 & 17 \\
\hline 5 & 2 & 2 & 1 & 2 \\
\hline 6 & 1 & 1 & 4 & 2 \\
\hline 6 & 2 & 0 & 4 & 2 \\
\hline 6 & 2 & 1 & 3 & 1 \\
\hline 1.617 & 0.309 & 1.215 & 0.093 & 66158 \\
\hline
\end{tabular}

Acknowledgements. The authors thank Masha Vlasenko for hosting and the American Institute of Mathematics for funding an LMFDB workshop to facilitate this work, and also thank William Stein for advising us on the use of Google's compute engine and Jan Steffen Müller for making us aware of the paper of Berry [2]. Finally, the authors thank the anonymous referees for their comments and corrections. 


\section{References}

1. M. H. Baker, E. GonzÁlez-Jiménez, J. GonzÁlez and B. Poonen, 'Finiteness results for modular curves of genus at least 2', Amer. J. Math. 127 (2005) 1325-1387.

2. T. G. BerRy, 'On periodicity of continued fractions in hyperelliptic function fields', Arch. Math. 55 (1990) 259-266.

3. B. J. Birch and W. KUYK (eds), Modular functions of one variable IV, Lecture Notes in Mathematics 476 (Springer, Berlin, 1975).

4. A. R. Booker, 'Numerical tests of modularity' J. Ramanujan Math. Soc. 20 (2005) no. 4, 283-339.

5. W. Bosma, J. Cannon, C. Fieker and A. Steel (eds), Handbook of Magma functions, edition 2.16 (2010) 5017 pages.

6. W. Bosma, J. Cannon and C. Playoust, 'The Magma algebra system I: The user language', J. Symbolic Comput. 24 (1997) 235-265.

7. A. Brumer and K. Kramer, 'The conductor of an abelian variety', Compositio Math. 92 (1994) $227-248$.

8. A. Brumer and K. Kramer, 'Paramodular abelian varieties of odd conductor', Trans. Amer. Math. Soc. 366 (2014) 2463-2516.

9. I. I. Bouw and S. Wewers, 'Computing L-functions and semistable reduction of superelliptic curves', Preprint, 2014, arXiv:1211.4459v4.

10. G. Cardona, E. Nart and J. Pujolas, 'Curves of genus two over fields of even characteristic', Math. Z. 250 (2005) 177-201.

11. G. Cardona and J. Quer, 'Field of moduli and field of definition for curves of genus 2', Computational aspects of algebraic curves, Lecture Notes Series on Computing 13 (World Scientific, Hackensack, NJ, 2005) 71-83.

12. J. E. Cremona, Algorithms for modular elliptic curves, 2nd edn (Cambridge University Press, Cambridge, 1997).

13. J. E. Cremona, 'The elliptic curve database for conductors to 130000', Algorithmic number theory: 7 th international symposium, ANTS-VII, Lecture Notes in Computer Science 4076 (eds F. Hess, S. Pauli and M. E. Pohst; Springer, Berlin, 2006) 11-29.

14. J. E. CRemona, 'Elliptic curve data', available at https://homepages.warwick.ac.uk/staff/J.E.Cremona/ $\mathrm{ftp} /$ data/.

15. T. Dokchitser, 'Computing special values of motivic L-functions', Exp. Math. 13 (2004) 137-149.

16. R. Dupont, 'Moyenne arithmético-géométrique, suites de Borchardt et applications', PhD Thesis, Laboratoire d'Informatique de l'École Polytechnique, 2006, available at http://www.lix.polytechnique.fr/ Labo/Regis.Dupont/these_soutenance.pdf.

17. D. Farmer, S. Koutsoliotas and S. Lemurell, 'Varieties via their L-functions', Preprint, 2015, arXiv:1502.00850v1.

18. F. Fité, K. S. Kedlaya, V. Rotger and A. V. Sutherland, 'Sato-Tate distributions and Galois endomorphism modules in genus 2', Compositio Math. 148 (2012) 1390-1442.

19. J. GonzÁlez, J. GuÀrdia and V. Rotger, 'Abelian surfaces of GL2-type as Jacobians of curves', Acta Arith. 116 (2005) 263-287.

20. J. GonzÁlez and V. Rotger, 'Equations of Shimura curves of genus two', Int. Math. Res. Not. IMRN 2004 (2004) no. 14, 661-674.

21. E. GonzÁlez-Jiménez and J. GonzÁlez, 'Modular curves of genus 2', Math. Comp. 72 (2002) $397-418$.

22. E. GonzÁlez-Jiménez, J. GonzÁlez and J. GuÀrdia, 'Computations on modular Jacobian surfaces', Algorithmic number theory: 5th international symposium, ANTS-V, Lecture Notes in Computer Science 2369 (eds C. Fieker and D. R. Kohel; Springer, Berlin, 2002) 189-197.

23. Google Inc., 'Google cloud platform', available at https://cloud.google.com.

24. D. Harvey and A. V. Sutherland, 'Computing Hasse-Witt matrices of hyperelliptic curves in average polynomial time', Algorithmic number theory (ANTS XI), LMS J. Comput. Math. 17 (2014) 257-273.

25. D. Harvey and A. V. Sutherland, 'Computing Hasse-Witt matrices of hyperelliptic curves in average polynomial time II', Frobenius distributions: Lang-Trotter and Sato-Tate conjectures, Contemporary Mathematics 663 (eds D. Kohel and I. E. Shparlinski; American Mathematical Society, Providence, RI, 2016), 127-148, to appear.

26. D. Holt, 'The meataxe as a tool in computational group theory', The atlas of finite groups: ten years on (Birmingham, 1995), London Mathematical Society Lecture Note Series 249 (Cambridge University Press, Cambridge, 1998) 74-81.

27. J.-I. Igusa, 'Arithmetic variety of moduli for genus two', Ann. of Math. (2) 72 (1960) 612-649.

28. F. Johansson, 'Arb: a C library for ball arithmetic', ACM Commun. Comput. Algebra 47 (2013) no. 4, $166-169$.

29. K. S. Kedlaya and A. V. Sutherland, 'Computing L-series of hyperelliptic curves', Algorithmic number theory: 8th international symposium, ANTS-VIII, Lecture Notes in Computer Science 5011 (eds A. J. van der Poorten and A. Stein; Springer, Berlin, 2008) 312-326.

30. A. K. Lenstra, H. W. Lenstra JR. and L. LovÁsz, 'Factoring polynomials with rational coefficients', Math. Ann. 261 (1982) 513-534. 
31. Q. Liu, 'Conducteur et discriminant minimal de courbes de genre 2', Compositio Math. 94 (1994) 51-79.

32. Q. Liu, 'Modèles minimaux des courbes de genre deux', J. reine angew. Math. 453 (1994) 137-164.

33. Q. Liu, 'Modèles entiers des courbes hyperelliptiques sur un corps valuation discrète', Trans. Amer. Math. Society 348 (1996) 4577-4610.

34. The LMFDB Collaboration, 'The $L$-functions and Modular Forms Database', available at http://www.lmfdb.org'.

35. P. Lockhart, 'On the discriminant of a hyperelliptic curve', Trans. Amer. Math. Soc. 342 (1994) 729-752.

36. J. R. Merriman and N. P. Smart, 'Curves of genus 2 with good reduction away from 2 with a rational Weierstrass point', Math. Proc. Cambridge Philos. Soc. 114 (1993) 203-214.

37. J.-F. Mestre, 'Construction de courbes de genre 2 à partir de leurs modules', Effective methods in algebraic geometry, Progress in Mathematics 94 (Birkhäuser, Boston, MA, 1991) 313-334.

38. The PARI-Group, PARI/GP version 2.7.0 Bordeaux, 2014, available at http://pari.math.u-bordeaux.fr/.

39. B. Poonen, 'Computational aspects of curves of genus at least 2', Algorithmic number theory (ANTS II), Lecture Notes in Computer Science 1122 (ed. H. Cohen; Springer, Berlin, 1996) 283-306.

40. Sage Developers, 'SageMath, the Sage Mathematics Software System (Version 7.2)', 2016, available at http://www.sagemath.org/.

41. J. SiJsling, 'Heuristic determination of endomorphism rings of curves', 2016, available at https://github.com/JRSijsling/heuristic_endomorphisms/.

42. N. P. Smart, 'S-unit equations, binary forms, and curves of genus 2', Proc. Lond. Math. Soc. (3) 75 (1997) $271-307$.

43. K. Soundararajan, 'Strong multiplicity one for the Selberg class', Canad. Math. Bull. 47 (2004) 468-474.

44. A. V. Sutherland, smalljac, version 4.1 .3 , available at http://math.mit.edu/ drew.

45. W. Stein and M. WAtKins, 'A database of elliptic curves-first report', Algorithmic number theory: 5th international symposium, ANTS- $V$, Lecture Notes in Computer Science 2369 (eds C. Fieker and D. R. Kohel; Springer, Berlin, 2002) 267-275.

46. M. Stoll, Genus 2 curve data files, at http://www.mathe2.uni-bayreuth.de/stoll/data/'.

47. P. B. VAN WAMELEN, 'Computing with the analytic Jacobian of a genus 2 curve', Discovering mathematics with Magma, Algorithms and Computation in Mathematics 19 (Springer, Berlin, 2006) 117-135.

\section{Andrew R. Booker \\ School of Mathematics \\ University of Bristol \\ University Walk \\ Bristol BS8 1TW \\ United Kingdom}

andrew.booker@bristol.ac.uk

\author{
Andrew V. Sutherland \\ Department of Mathematics \\ Massachusetts Institute of Technology \\ 77 Massachusetts Avenue \\ Cambridge, MA 02139 \\ USA \\ drew@math.mit.edu
}

\author{
Dan Yasaki \\ Department of Mathematics and \\ Statistics \\ University of North Carolina at \\ Greensboro \\ 317 College Avenue \\ Greensboro, NC 27412 \\ USA \\ d_yasaki@uncg.edu
}

\author{
Jeroen Sijsling \\ Department of Mathematics \\ Dartmouth College \\ 6188 Kemeny Hall \\ Hanover, NH 03755 \\ USA
}

sijsling@gmail.com

John Voight
Department of Mathematics
Dartmouth College
6188 Kemeny Hall
Hanover, NH 03755
USA
jvoight@gmail.com

John Voight

Department of Mathematics

Dartmouth College

6188 Kemeny Hall

USA

jvoight@gmail.com 